\title{
CONTROL OF A ONE RIGIT-LINK MANIPULATOR IN THE CASE OF NONSMOOTH TRAJECTORY
}

\author{
N.K. Aksenova, V.A. Sobolev \\ Samara National Research University, Samara, Russia
}

\begin{abstract}
Mathematical model of a single-link manipulator is considered. It describes the motion of the manipulator along a given path which cam be nonsmooth. Integral manifolds method is used for the system order reduction and design of control laws.
\end{abstract}

Keywords: single-link manipulator, integral manifold, singular perturbances.

Citation: Aksenova N, Sobolev V. Control of a one rigit-link manipulator in the case of nonsmooth trajectory. CEUR Workshop Proceedings, 2016; 1638: 493-497. DOI: 10.18287/1613-0073-2016-1638-493-497

\section{Single-link manipulator model}

The simplest model of the manipulator consists of one link. In this case the equations of the manipulator motions are

$J_{1} \ddot{q}_{1}+M g l \sin q_{1}+c\left(\dot{q}_{1}-\dot{q}_{m}\right)+k\left(q_{1}-q_{m}\right)=0$,

$J_{m} \ddot{q}_{m}-c\left(\dot{q}_{1}-\dot{q}_{m}\right)-k\left(q_{1}+q_{m}\right)=u$,

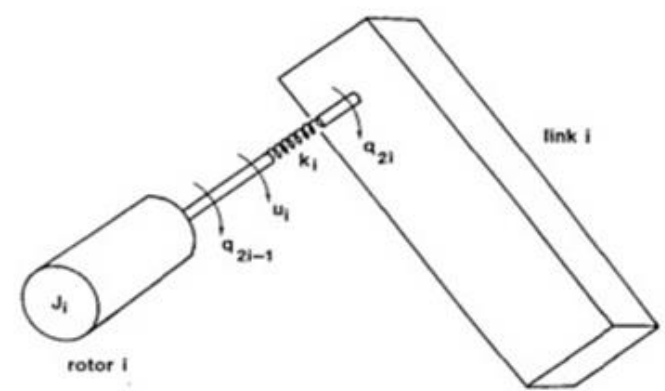

Fig. 1. Single-link manipulator model 
where: $J_{m}$-is the motor inertia; $J_{1}$-is the link inertia; $M$-is the link mass, $l-$ is the link length; $c$-is the damping coefficient; $k-$ is the stiffness; $q_{1}-$ is the link angle; $q_{m}$-is the $\mathrm{r}$ angle, and $u$ is the torque input which is the controller.

The use of the small parameter $\varepsilon=\frac{1}{\sqrt{k}}$ and new variables

$x_{1}=\frac{J_{1} q_{1}+J_{m} q_{m}}{J_{1}+J_{m}}, \quad x_{2}=\dot{x}_{1}, y_{1}=q_{1}-q_{m}, y_{2}=\varepsilon \dot{y}_{1}$,

yields the system

$\dot{x}_{1}=x_{2}, \quad \dot{x}_{2}=\frac{M g l}{J_{1}+J_{m}} \sin \left(x_{1}+\frac{J_{m}}{J_{1}+J_{m}} y_{1}\right)+\frac{u}{J_{1}+J_{m}}$

$\varepsilon \dot{y}_{1}=y_{2}, \varepsilon \dot{y}_{2}=-\left(\frac{1}{J_{1}}+\frac{1}{J_{m}}\right) y_{1}-\varepsilon c\left(\frac{1}{J_{1}}+\frac{1}{J_{m}}\right) y_{2}-\varepsilon^{2} \frac{M g l}{J_{1}} *$

$\sin \left(x_{1}+\frac{J_{m}}{J_{1}+J_{m}} y_{1}\right)-\varepsilon^{2} \frac{u}{J_{m}}$.

This system is singularly perturbed with slow subsystem (3) and fast subsystem (4). Neglecting all terms of order $O\left(\varepsilon^{2}\right)$ in the right hand side of the last equation the independent subsystem is obtained.

$\varepsilon \dot{y}_{1}=y_{2}, \varepsilon \dot{y}_{2}=-\left(\frac{1}{J_{1}}+\frac{1}{J_{m}}\right) y_{1}-\varepsilon c\left(\frac{1}{J_{1}}+\frac{1}{J_{m}}\right) y_{2}$,

Solutions of which are characterized by high frequency

$\frac{\sqrt{\left(\frac{1}{J_{1}}+\frac{1}{J_{m}}\right)}}{\varepsilon}$

and relatively slow decay $\frac{c\left(\frac{1}{J_{1}}+\frac{1}{J_{m}}\right)}{2}$, since this differential system has a characteristic polynomial

$\varepsilon^{2} \lambda^{2}+\varepsilon c\left(\frac{1}{J_{1}}+\frac{1}{J_{m}}\right) \lambda+\left(\frac{1}{J_{1}}+\frac{1}{J_{m}}\right)$

with complex zeros

$\lambda_{1,2}=-\frac{c}{2}\left(\frac{1}{J_{1}}+\frac{1}{J_{m}}\right) \pm \frac{i}{2 \varepsilon} \sqrt{\left(\frac{1}{J_{1}}+\frac{1}{J_{m}}\right)-\varepsilon^{2} \frac{c^{2}}{4}\left(\frac{1}{J_{1}}+\frac{1}{J_{m}}\right)^{2}}$

Since the real part of these numbers is negative, slow invariant manifold can be used for the analysis of the manipulator model under consideration noting that this manifold is attractive and the reducibility principle holds [1]. Note that the Jacobian matrix of fast subsystem (4) for $\varepsilon=0$ has eigenvalues on the imaginary axis with nonvan- 
ishing imaginary parts. This means that we have so called critical case [1, 6]. A similar case and some other critical cases have been investigated in [7-12].

\section{Slow integral manifold}

The terms of $O\left(\varepsilon^{2}\right)$ of the fast subsystem (4) leads us to conclude that the slow integral manifold may be found in the form $y_{1}=\varepsilon^{2} Y+O\left(\varepsilon^{3}\right)$ and $y_{2}=O\left(\varepsilon^{3}\right)$, where

$Y=-\left[\frac{M g l}{J_{1}} \sin \left(x_{1}\right)+\frac{u_{0}}{J_{m}}\right]\left(\frac{1}{J_{1}}+\frac{1}{J_{m}}\right)^{-1}$

Here we used the representation

$u=u_{0}+\varepsilon^{2} u_{1}+O\left(\varepsilon^{3}\right)$.

The flow on this manifold is described by equations:

$\dot{x}_{1}=x_{2}, \quad \dot{x}_{2}=-\frac{M g l}{J_{1}+J_{m}} \sin \left(x_{1}+\varepsilon^{2} \frac{J_{m}}{J_{1}+J_{m}} Y\right)+\frac{u_{0}+\varepsilon^{2} u_{1}}{J_{1}+J_{m}}+O\left(\varepsilon^{3}\right)$

Note that due to (2) $q_{1}$ is expressed through new variables

$q_{1}=x_{1}+\frac{J_{m}}{J_{m}+J_{1}} y_{1}$

where

$y_{1}=\varepsilon^{2} Y+O\left(\varepsilon^{3}\right)$

what allows presenting the system (5) on the slow integral manifold as

$\ddot{q}_{1}-\varepsilon^{2} \frac{J_{m}}{J_{m}+J_{1}} \ddot{Y}=-\frac{M g l}{J_{1}+J_{m}} \sin \left(q_{1}\right)+\frac{u_{0}+\varepsilon^{2} u_{1}}{J_{1}+J_{m}}+O\left(\varepsilon^{3}\right)$

\section{Control function}

Assume $q_{d}$ is the desired trajectory of the manipulator movement. Unlike $[4,5]$ we do not use a fast term added to the control input to make the fast dynamics asymptotically stable to guarantee the fast decay of fast variables $y_{1}, y_{2}$. We use the slow component of the control function $u$ which is written as a sum.

$u_{0}=\left(J_{1}+J_{m}\right) u_{d}+M g l \sin q_{1}$

where

$u_{d}=\ddot{q}_{d}-a_{1}\left(x_{1}+q_{d}\right)-a_{2}\left(\dot{x}_{1}+\dot{q}_{d}\right)$.

Setting $\varepsilon=0$, using (7) and the definition of $u_{0}$ and $u_{d}$ we obtain to an accuracy of order $O\left(\varepsilon^{2}\right)$

$\ddot{q}_{1}-\ddot{q}_{d}+a_{2}\left(\ddot{q}_{1}+\ddot{q}_{d}\right)+a_{1}\left(q_{1}+q_{d}\right)=0$

Information Technology and Nanotechnology (ITNT-2016) 
for the difference $q_{1}-q_{d}$, since $q_{1}=x_{1}+O\left(\varepsilon^{3}\right)$ on the slow integral manifold.

Equation (8) gives the possibility to choose coefficients in the control function $u_{d}$ in such a way that the corresponding control function gives the possibility of realizing a desired trajectory. Assume, for example [4, 5], $M=1, k=100, l=1, J_{1}=1, J_{m}=1, g=9.8$, $c=2$. Setting $a_{1}=3, a_{2}=4$ for the desired trajectory $q_{d}=\sin t$ we obtain the following control law for the original variables

$u=2 u_{d}+9.8 \sin \left(q_{1}\right)=2\left[-\sin t-4\left(\dot{q}_{1}-\cos t\right)-3\left(q_{1}-\sin t\right)\right]+9.8 \sin \left(q_{1}\right)$

It is illustrated in fig. 2 that the trajectory of controlled single-link manipulator tends to the desired trajectory $q_{d}$ as $t$ increases.

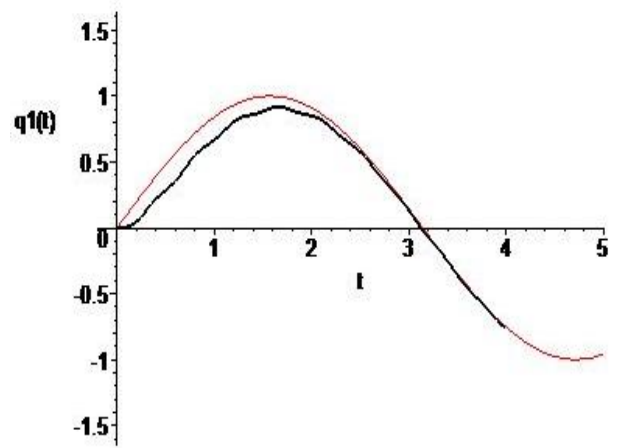

Fig. 2. The graph of the desired trajectory (red line) and the trajectory of controlled single-link manipulator (black line)

In many cases manipulators describe nonsmooth paths, polygonal lines, for example. It is impossible to use the integral manifolds method to construct approximations of slow integral manifold as an expansions in powers of the small parameter. One possibility is the use of polynomial smoothing.

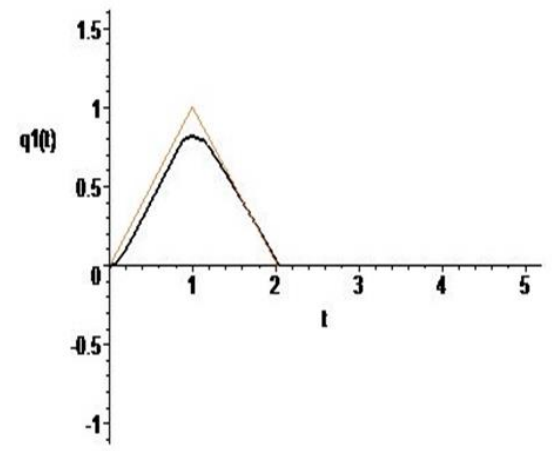

Fig. 3. The graph of the desired trajectory (red line) and the trajectory of controlled single-link manipulator (black line) 
It is illustrated in fig. 3, which contains the response of the controlled single-link manipulator, that the trajectory tends to the desired polygonal trajectory $q_{d}$ to the approximation of which is

$q_{d}=\left\{\begin{array}{c}x, 0<x<\delta-1 \\ a(x-1)^{4}+b(x-1)^{2}+1, \delta-1<x<\delta+1 \\ -x+2, \delta+1<x<2\end{array}\right.$

\section{Conclusion}

The manipulator model describing the manipulator motion in a nonsmooth path is considered. Integral manifold method is used for the system order reduction.

\section{Acknowledgment}

This work is supported in part by the Russian Foundation for Basic Research (grant 14-01-97018-p) and the Ministry of Education and Science of the Russian Federation under the Competitiveness Enhancement Program of Samara University (2013-2020).

\section{References}

1. Shchepakina EA, Sobolev VA, Mortell MP. Singular Perturbations. Introduction to system order reduction methods with applications, 2014; $121 \mathrm{p}$.

2. Sobolev VA, Shchepakina EA. Models Reduction and Critical Phenomena in Macrokinetics. M.: Fizmatlit, 2010.

3. $\quad$ Li EB, Marcus L. Optimal Control Fundamental. M.: Nauka, 1972; 576 p.

4. Ghorbel, F, Spong, MW. Integral manifolds of singularly perturbed systems with application to rigid-link flexible-joint multibody systems. Int. J. of Non-Linear Mechanics, 2000; 35: 133-155.

5. Spong, MW. Modeling and control of elastic joint robots. J. of Dynamic Systems, Measurement and Control, 1987; 109(4): 310-319.

6. Vasilieva AB, Butuzov VF. Singularly Perturbed Equations in the Critical Case Tech. Report MRC TSR. Madison: University of Visconsin, 1980: 2039.

7. Gu Z, Nefedov NN, O'Malley RE. On singular singularly perturbed initial values problems SIAM J Appl Math, 1989: 49: 1.

8. Kononenko LI, Sobolev VA. Asymptotic expansion of slow integral manifolds Siberian Math J, 1994; 35: 1119-1132.

9. Shchepakina EA. Two Forms of Stability Change for Integral Manifolds. Differential Equations, 2004; 40(5): 766-769.

10. Shchepakina E. Canards and black swans in model of a 3-D autocatalator. Journal of Physics: Conference Series, 2005; 22: 194-207.

11. Shchepakina E, Korotkova O. Condition for canard explosion in a semiconductor optical amplifier. Journal of the Optical Society of America B: Optical Physics, 2011; 28(8): 1988-1993.

12. Shchepakina E, Korotkova O. Canard explosion in chemical and optical systems. Discrete and Continuous Dynamical Systems. Series B, 2011; 18(2): 495-512. 